\title{
Treatment of Acute Vertebrobasilar Occlusion Using Thrombectomy with Stent Retrievers: Initial Experience with 18 Patients
}

\author{
M. Espinosa de Rueda, G. Parrilla, J. Zamarro, B. García-Villalba, F. Hernández, and A. Moreno
}

\begin{abstract}
BACKGROUND AND PURPOSE: Acute vertebrobasilar occlusion is an ominous disease with few proved effective treatments. Experience with stent retrievers is scarce and limited to combined therapies (stent retrievers associated with previous intravenous fibrinolysis, intra-arterial thrombolysis, or other mechanical devices). We present our experience with 18 patients treated with direct thrombectomy by using stent retrievers.

MATERIALS AND METHODS: Eighteen patients with vertebrobasilar occlusion were treated with direct thrombectomy by using stent retrievers at our hospital. The mean age was 67.5 years. Clinical presentation was sudden deterioration in consciousness level in $61.2 \%$ and progressive or fluctuating brain stem symptoms in 38.8\%. Stroke subtype (TOAST) was atherothrombotic (33.3\%), undetermined (33.3\%), cardioembolic (27.7\%), and of unusual etiology (5.5\%).

RESULTS: The occlusion site was the vertebral artery in 1 case, proximal basilar artery in 4, middle basilar artery in 6, distal basilar artery in 5 , and unilateral posterior cerebral artery in 2 cases. SRs included the Solitaire AB in 8 cases, Solitaire FR in 5 cases, and Trevo Pro in 5 cases. An 8F Merci balloon guide catheter was used in 15 patients, and a Neuron 6F, in 3 patients. Post-clot retrieval definitive intracranial stents were used in 5 patients (27.7\%). Postprocedural $\mathrm{TICl} \geq 2 \mathrm{~b}$ was achieved in 17 patients (94.4\%). Clinically, $72.2 \%$ of patients experienced an improved NIHSS score at discharge, $22.2 \%$ died, and in 5.5\% the NIHSS scores did not change. The mRS score at 3 months was $0-2$ in 9 patients $(50 \%)$ and $3-5$ in 5 patients $(27.7 \%)$.
\end{abstract}

CONCLUSIONS: Thrombectomy with stent retrievers is feasible in the treatment of vertebrobasilar occlusion. These initial results must be confirmed by further prospective studies with a larger number of cases.

ABBREVIATIONS: $\mathrm{mRS}=$ modified Rankin Scale; $\mathrm{SR}=$ stent retriever; $\mathrm{TICI}=$ Thrombolysis in Cerebral Infarction; TOAST = Trial of Org 10172 in Acute Stroke Treatment; $\mathrm{VA}=$ vertebral artery

A cute vertebrobasilar occlusion is generally associated with an ominous prognosis, ${ }^{1}$ with early recanalization being the only factor that seems to be associated with a favorable clinical outcome. ${ }^{2,3}$ Antithrombotic agents and intravenous or intra-arterial thrombolytics have classically yielded poor results in achieving recanalization of these vessels, ${ }^{4-11}$ but in the past few years, the

Received June 7, 2012; accepted after revision August 2.

From the Department of Interventional Neuroradiology (M.E.d.R., G.P., J.Z., B.G.-V., F.H., A.M.) and Department of Neurology (G.P.), Hospital Universitario Virgen de la Arrixaca, Murcia, Spain.

Mariano Espinosa de Rueda, Guillermo Parrilla, Joaquín Zamarro, and Antonio Moreno are participating in the Solitaire FR Thrombectomy for Acute Revascularization in Anterior Circulation (STAR) trial. Our study includes only posterior circulation strokes, which is not the aim of the STAR trial. However, we used Solitaire FR in 5 of our 18 patients.

Please address correspondence to Mariano Espinosa de Rueda, MD, Department of Interventional Neuroradiology, Hospital Universitario Virgen de la Arrixaca, Carretera Murcia-Cartagena, S/N, El Palmar, Murcia, Spain, 30100; e-mail: mm.espinosa@ gmail.com, nrxhuva@gmail.com.

http://dx.doi.org/10.3174/ajnr.A3329 appearance of mechanical thrombectomy devices for cerebral circulation, especially stent retrievers, seems to have opened a new window for the effective treatment of vertebrobasilar occlusion and other large-vessel stroke. ${ }^{12-22}$ However, experience with stent retrievers in this disease seems to be quite limited in recent medical literature and restricted to combined therapy (associated with intravenous and/or intra-arterial thrombolysis). In this article, we present the results in our hospital of 18 consecutive cases of vertebrobasilar occlusion that were treated with thrombectomy by using stent retrievers.

\section{MATERIALS AND METHODS}

This series included a retrospective analysis of all patients who presented at our hospital with acute vertebrobasilar stroke and were treated directly with intra-arterial thrombectomy by using retrievable stents between January 2010 and January 2012.

All patients with stroke who arrive at our hospital undergo an initial imaging protocol that includes urgent nonenhanced CT, 
CT angiography, and CT perfusion. Patients with an NIHSS score of $>6$ and evidence of vertebrobasilar occlusion on CT angiography undergo mechanical thrombectomy in the initial 24-hour period after stroke onset. For patients who are referred from other centers, we recommend administration of intravenous thrombolysis during patient transfer and angiography suite preparation. The exclusion criteria for mechanical thrombectomy are the presence of cerebral hemorrhage, evidence of established large cerebellar and brain stem infarction on nonenhanced CT, the absence of vascular occlusion on CT angiography, $>24$ hours since symptom onset, or any medical conditions that preclude general anesthesia. Before angiography and/or mechanical thrombectomy, all patients (or relatives) must give informed consent.

All thrombectomy procedures in these series were performed with the patient under general anesthesia. During the procedure, no antiplatelet or intravenous anticoagulants were administered (except heparin in the flushing systems). Angiography was performed via femoral approach by using a 9F sheath and a diagnostic catheter, generally a JB 4F (Cordis, Miami Lakes, Florida). An initial angiographic series from both vertebral arteries was performed to confirm occlusion and to evaluate the best access to the occlusion site. Location of the clot was divided into the vertebral artery, the proximal basilar artery (from the confluence of the vertebral arteries to the anterior inferior cerebellar artery), the mid-basilar artery (from the anterior inferior cerebellar artery to the superior cerebellar artery), the distal basilar artery (distal to the superior cerebellar artery), and the posterior cerebral artery.

Once the location of the clot had been identified, the diagnostic catheter was removed and an $8 \mathrm{~F}$ Merci balloon guide catheter (Concentric Medical, Mountain View, California) was advanced and placed in the cervical segment of the dominant vertebral artery. In cases in which this balloon guide catheter could not be placed in the vertebral artery due to the small caliber of the vessel, a Neuron 6F delivery catheter (Penumbra, Alameda, California) was placed instead. A Rebar-18, Marksman-21 (both ev3, Irvine, California), or Trevo-18 microcatheter (Concentric Medical) with a 0.014 -inch microwire (generally a Transend [Boston Scientific, Natick, Massachusetts] or Traxcess [MicroVention, Aliso Viejo, California]) was then advanced distally to the clot. The microwire was then removed, and a stent retriever (either Solitaire AB, Solitaire FR [ev3], or Trevo) was used to engage and snare the clot. Once the clot was captured, the balloon guide catheter was inflated to temporarily arrest forward flow while the clot was being withdrawn. The clot was first pulled into the guide catheter and then completely out of the body while aspirating with a 50 -mL syringe. The balloon was then deflated, and the flow was restored. In cases performed with Neuron as the guide catheter, retrieval of the stent and aspiration were performed without vessel occlusion. In the event of major residual stenosis due to an atherosclerotic plaque, a definitive intracranial stent (Pharos Vitesse; Micrus Endovascular, San Jose, California) was deployed. All these patients received a loading dose of $300 \mathrm{mg}$ of clopidogrel (by a nasogastric tube) and $500 \mathrm{mg}$ of intravenous aspirin (lysine acetylsalicylate) before placement of the definitive stent.

After the procedure, patients were admitted to the intensive care unit. A nonenhanced CT was routinely performed in the first 24 hours after the procedure to rule out hemorrhage, and anti- platelet or anticoagulation therapy was then initiated. The choice of therapy depended on the suspected etiology of the stroke. Initial anticoagulation with intravenous heparin and a posterior switch to acenocoumarol was used for inferred cardioembolism, while antiplatelet therapy (aspirin, $200 \mathrm{mg}$ a day, or clopidogrel, $75 \mathrm{mg}$ a day) was preferred when atherothrombotic etiologies were suspected. The control of blood pressure, fever, hyperglycemia, aspiration risk, deep venous thrombosis prophylaxis, and all other essential aspects in acute stroke management was carried out following current stroke guidelines. ${ }^{23}$

Clinical data recorded for the study included sex, age, vascular risk factors (high blood pressure, diabetes mellitus, hypercholesterolemia, cigarette smoking, atrial fibrillation, previous stroke, coronary disease), stroke subtype according to the TOAST classification, ${ }^{24}$ occluded artery, and initial NIHSS and mRS scores. The outcome measures included arterial recanalization according to the Thrombolysis in Cerebral Infarction scale, both postprocedural and discharge NIHSS scores, and discharge mRS and 90-day mRS scores.

Extensive additional technical data from each procedure were also obtained, including the type of guide catheter, the artery used for guide-catheter placement, the occlusion site, the type of retrievable stent used, the number of devices needed for retrieval, the number of passes, the definitive intracranial stent, procedural time, the time from symptom onset to groin puncture, and also the time to revascularization.

\section{RESULTS}

Between January 2010 and January 2012, twenty-one patients with vertebrobasilar occlusion were treated with mechanical thrombectomy at our center. Three were referred from outside hospitals and had been previously treated with intravenous thrombolysis. These 3 patients were excluded from our study. The remaining 18 patients came straight to our center and were directly treated with mechanical thrombectomy. Our article analyzes the results in this group of patients.

\section{Baseline Characteristics}

Seven patients were men, and 11, women. The mean age was 67.5 years, ranging from 32 to 87 years. Hypertension was the most prevalent risk factor $(77.5 \%)$, followed by dysphemia (50\%), diabetes mellitus (33.3\%), a smoking habit $(27.7 \%)$, and atrial fibrillation (27.7\%). Before stroke onset, 5 patients were being treated with antiaggregates (aspirin or clopidogrel), due to a previous stroke $(n=3)$, atrial fibrillation $(n=1)$, and cardiovascular risk factors $(n=1)$. Two were taking acenocoumarol because of atrial fibrillation, 1 was on low-molecular-weight heparin due to a previous pulmonary thromboembolism, and 1 patient was on both antiplatelet and oral anticoagulation due to coronary disease and a metallic mitral valve. The stroke subtype (according to the TOAST classification) was atherothrombotic in 6 patients $(33.3 \%)$, cardioembolic in $5(27.7 \%)$, undetermined in $6(33.3 \%)$, and of unusual etiology in $1(5.5 \%)$.

\section{Treatment}

Grade and Site of Occlusion. Sixteen patients (88.8\%) showed complete occlusion of the affected vessel (TICI 0). Patients 4 and

AJNR Am J Neuroradiol 34:1044-48 May 2013 www.ajnr.org 1045 
18 had TICI 1. The occlusion site was the intracranial vertebral artery in 1 patient $(5.5 \%)$, the proximal basilar artery in 4 $(22.2 \%)$, the middle basilar artery in $6(33.3 \%)$, the distal basilar artery in $5(27.7 \%)$, and the posterior cerebral artery in 2 patients $(11.1 \%)$.

Catheter Guide Type and Placement. In 15 patients (83.3\%), an $8 \mathrm{~F}$ balloon guide catheter was placed in the cervical segment of the dominant vertebral artery, allowing posterior vessel occlusion and aspiration during retrieval. This approach was not possible in the remaining 3 patients (16.7\%) due to the small caliber of the vertebral artery; a 6F Neuron guide catheter was placed instead. Control angiograms did not show dissection or vessel damage in the segments used for the placement of the guide catheters. Two patients presented with induced mechanical vasospasm during the procedure, which was resolved completely by relocating the catheter.

Procedure Times and Devices. The mean time from stroke onset to groin puncture was 365.9 minutes. The mean procedure time was 75.1 minutes. The mean time to recanalization was 441.1 minutes. The mean number of passes per procedure was 1.7.

The Solitaire AB $4 \times 15$ was used in 2 patients (patients 3 and 12); the Solitaire $A B 4 \times 20$, in 7 patients (patients 1, 2, 3, 5, 6, 7, 9); the Solitaire AB $6 \times 20$, in 1 patient (patient 4); the Solitaire FR $4 \times 15$, in 1 patient (patient 12); the Solitaire FR $4 \times 20$, in 4 patients (patients $11,15,16$, and 18 ); the Solitaire FR $6 \times 30$, in 1 patient (patient 11); and the Trevo Pro $4 \times 20$, in 5 patients (patients 8, 10, 13, 14, and 17).

We considered associated treatment with definitive intracranial stent placement necessary in 5 patients (27.7\%): patients 2 (Pharos Vitesse $2.5 \times 13$ ), 3 (Pharos Vitesse $3.5 \times 18$ ), 9 (Pharos Vitesse $3.0 \times 15), 11($ Pharos Vitesse $2.25 \times 10,2.5 \times 13,4.0 \times$ 18 ), and 15 (Pharos Vitesse $3.5 \times 18$ ).

Recanalization Rates and Complications. Good recanalization rates (TICI $\geq 2 \mathrm{~b}$ ) were achieved in 17 of the 18 patients $(94.4 \%$ ). TICI 3 was obtained in 14 patients $(77.7 \%)$; TICI $2 \mathrm{~b}$, in 3 patients (16.6\%); and TICI 1 , in 1 patient $(5.5 \%)$.

Two of the cases with TICI $2 \mathrm{~b}$ (patients 3 and 5) corresponded to distal small vessel occlusions that were observed after the retrieval of the thrombus. The remaining TICI $2 \mathrm{~b}$ case (patient 12 ) was due to a stenotic atherosclerotic plaque in the $\mathrm{P} 3$ segment of the right posterior cerebral artery, which caused a distal partial perfusion deficit.

The TICI 1 case corresponded to patient 11, who presented with severe stenosis of the right vertebral artery ostium associated with a proximal basilar artery occlusion. In this patient, revascularization could not be achieved despite the use of 2 different stent retrievers (Solitaire FR $4 \times 20$ and $6 \times 30$ ) and the deployment of 3 definitive stents ( 2 in the basilar artery and 1 in the right vertebral artery ostium).

Procedure-related complications were not observed in any patients. In patient 5, a focal subarachnoidal hemorrhage was observed on the control CT performed 24 hours after the procedure, though no vascular rupture or contrast extravasation was observed on imaging during the intervention. We interpreted this case as a reperfusion phenomenon.

\section{Clinical Evolution}

All patients had an mRS score between 0 and 2 before the stroke. Eleven patients $(61.2 \%)$ experienced a sudden deterioration in the level of consciousness as the initial symptom, and the remainder of the patients $(38.8 \%)$ presented with progressive or fluctuating brain stem deficiency symptoms. Four of the patients $(22.2 \%)$ arrived at the hospital already intubated. The mean NIHSS score on arrival in the nonintubated patients was 20.4. Thirteen of the 18 patients (61.2\%) experienced an NIHSS improvement at discharge, ranging from 4 to 25 points (mean, 14 points). In 1 patient (patient 5), the NIHSS score did not change, and 4 patients died $(22.2 \%)$. The mean NIHSS at discharge was 6.4 points.

The mRS score at discharge was $0-2$ in 7 patients $(38.8 \%)$ and $3-5$ in 7 patients $(38.8 \%)$, and the remaining 4 patients died $(22.2 \%)$. Three months after treatment, the mRS score was $0-2$ in 9 cases $(50 \%)$ and $3-5$ in 5 cases $(27.7 \%)$.

\section{DISCUSSION}

Acute vertebrobasilar occlusion is an uncommon cause of stroke, which has classically been associated with a poor clinical outcome. ${ }^{1}$ Several studies have proved that the only relevant factor in achieving a good prognosis in this disease is early recanalization, ${ }^{2,3}$ but treatment with antiplatelet or anticoagulant agents has yielded very low recanalization rates, and intravenous thrombolysis has repeatedly been shown to be poorly effective in the recanalization of vertebrobasilar occlusion or other large vessels such as the internal carotid artery or the proximal middle cerebral artery. ${ }^{4-11}$

In the past few years, mechanical thrombectomy devices, especially stent retrievers, have proved to be a new option in the treatment of large-vessel stroke, achieving higher recanalization percentages and better clinical outcomes than previous treatments. ${ }^{12-22}$ However, experience with stent retrievers in vertebrobasilar occlusion in the medical literature seems to be quite scarce and includes mainly cases treated with combined therapy (initial intravenous fibrinolysis until the angiographic suite is prepared, followed by thrombectomy with stent retrievers and continuation of the intravenous recombinant tissue plasminogen activator during the endovascular procedure), associated intra-arterial thrombolysis, and/or concomitant use of other mechanical devices.

In the most recent related study, Mordasini et $\mathrm{al}^{21}$ used a multimodal treatment that consisted of a combination of thromboaspiration, intravenous and/or intra-arterial thrombolysis, mechanical thrombectomy, and percutaneous angioplasty/stent placement. Andersson et $\mathrm{al}^{22}$ examined 28 cases of vertebrobasilar occlusion treated with different mechanical devices (including stent retrievers and/or the Merci retriever [Concentric Medical]) and supplemental therapies such as intra-arterial recombinant tissue plasminogen activator, balloon angioplasty, intra-arterial nicardipine, or low-molecular-weight heparin. In a previous study, Costalat et $\mathrm{al}^{16}$ analyzed 16 cases of basilar occlusion that were treated with combined therapy, as part of a multidisciplinary protocol (RECOST [Rescue, Combined and Stand-Alone Thrombectomy] study) that selected from 3 treatment strategies for large-vessel occlusions (middle cerebral artery, internal carotid artery, and basilar artery), according to the time of symptom 
Review of published reports about recanalization and outcome of patients with acute vertebrobasilar occlusions treated with thrombolysis and/or thrombectomy

\begin{tabular}{|c|c|c|c|c|c|c|}
\hline Reference & Treatment & $\begin{array}{l}\text { No. of } \\
\text { Patients }\end{array}$ & $\mathrm{TICl} 2-3$ & 90-Day mRS 0-2 & Mortality & ICH \\
\hline Sairanen et $\mathrm{al}^{10}$ & IVT & 116 & $64.8 \%$ & $26 \%$ & $41.4 \%$ & $15.7 \%$ \\
\hline Lindsberg and Mattle ${ }^{8}$ & IVT & 76 & $53 \%$ & $22 \%$ & $52.6 \%$ & $11 \%$ \\
\hline Lindsberg and Mattle ${ }^{8}$ & IAT & 344 & $65 \%$ & $24 \%$ & $55.2 \%$ & $8 \%$ \\
\hline Schulte-Altedorneburg et al ${ }^{9}$ & IAT & 180 & $55 \%$ & $26.5 \%$ & $43 \%$ & $32 \%$ \\
\hline Lutsep et $\mathrm{al}^{19}$ & IVT \pm Merci \pm IAT & 27 & $78 \%$ & $33 \%$ & $44 \%$ & $19 \%$ \\
\hline Pfefferkorn et $\mathrm{al}^{20}$ & $\mathrm{IVT} \pm$ Merci $\pm \mathrm{TA}$ & 26 & $85 \%$ & $38 \%$ & $31 \%$ & $8 \%$ \\
\hline Miteff et $\mathrm{al}^{18}$ & $\mathrm{SR} \pm \mathrm{IAT} \pm \mathrm{TA}$ & 10 & $100 \%$ & $20 \%$ & $33.3 \%$ & $10 \%$ \\
\hline Costalat et $\mathrm{al}^{16}$ & $\mathrm{IVT}+\mathrm{SR}$ & 16 & $81 \%$ & $44 \%$ & $25 \%$ & $6.2 \%$ \\
\hline Andersson et $\mathrm{al}^{22}$ & Merci $\pm \mathrm{IAT} \pm \mathrm{SR}$ & 28 & $96 \%$ & $57 \%$ & $21 \%$ & $10.7 \%$ \\
\hline Mordasini et $\mathrm{al}^{21}$ & $\mathrm{IVT} \pm \mathrm{IAT} \pm \mathrm{TA} \pm \mathrm{SR}$ & 14 & $100 \%$ & $28.6 \%$ & $35.7 \%$ & $21.4 \%$ \\
\hline Our group & $S R$ & 18 & $94.4 \%$ & $50 \%$ & $22.2 \%$ & $5.5 \%$ \\
\hline
\end{tabular}

Note:-IAT indicates intra-arterial thrombolysis; ICH, intracranial hemorrhage; IVT, intravenous thrombolysis; Merci, Merci retriever; TA, thromboaspiration.

onset and the location of vessel occlusion. Machi et al ${ }^{17}$ treated 15 patients with vertebrobasilar occlusion between 0 and 24 hours after symptom onset with the same strategy (combined therapy) in a study designed to test the Solitaire FR in large-vessel disease of both the anterior and posterior circulation. Miteff et al ${ }^{18}$ treated 8 vertebrobasilar occlusions with the Solitaire $\mathrm{AB}$ and subsequent thrombectomy with the Penumbra system (Penumbra, Alameda, California) and/or intra-arterial urokinase if successful recanalization was not achieved.

In this series, we report the effective treatment of 18 vertebrobasilar occlusions at our center by using thrombectomy with stent retrievers. Our percentage of successful recanalization $(94.4 \%)$ is clearly higher than that obtained with intravenous/intra-arterial thrombolysis $^{8-11}$ or a Merci retriever ${ }^{19,20}$ and is similar to that obtained with stent retrievers and other coadjutant therapies ( $\mathrm{Ta}$ ble). ${ }^{16-18,21,22}$ The mRS score of 0-2 at 3 months in our series was $50 \%$, compared with $28.6 \%$ in the series of Mordasini et al, ${ }^{21} 57 \%$ in Andersson et al, ${ }^{22} 44 \%$ in Costalat et al, ${ }^{16}$ and 20\% in Miteff et al. ${ }^{18}$ We have observed a mortality rate of $22.2 \%$ in our patients, compared with $35.7 \%$ in the series of Mordasini et al, ${ }^{21} 21 \%$ in Andersson et $\mathrm{al}^{22}{ }^{22}$ 25\% in Costalat et $\mathrm{al}^{16}{ }^{16}$ and $33.3 \%$ in Miteff et al. ${ }^{18}$ Globally, these results seem to indicate that thrombectomy with stent retrievers is a feasible treatment for acute vertebrobasilar occlusion.

Our series also seems to support the idea that the mechanical disruption and retrieval of the clot with the stent retrievers is the key in revascularization of vertebrobasilar occlusions. This aspect might have previously been questioned due to the associated treatment in previous series (intravenous thrombolysis, intra-arterial urokinase, or other retrievers such as the Penumbra or the Merci retriever) and is congruent with the efficacy that thrombectomy with stent retrievers has shown in the treatment of other large-vessel occlusions. ${ }^{14-18}$

Some technical aspects of our series are also noteworthy. In our cases of vertebrobasilar occlusion, we were able to place an $8 \mathrm{~F}$ balloon-guided catheter in the cervical vertebral artery in 15 of 18 patients $(83.3 \%)$, despite the small size of the vertebral artery (in comparison with the internal carotid artery, the common location for the balloon guide catheter in anterior circulation strokes); on most occasions, its diameter is $>2.7 \mathrm{~mm}(8 \mathrm{~F})$, thus making this approach possible and complete occlusion and aspiration during retrieval feasible. Also of interest, the deployment of intracranial stents in postretrieval of residual stenosis did not seem to be associated with procedural complications or bad clinical results in our series, which may make this an interesting option in the treatment of residual lesions of atherothrombotic origin, which are evident after the initial retrieval of the clot. Studies with a larger number of patients are needed to confirm the safety and efficacy of this option.

\section{CONCLUSIONS}

Thrombectomy with stent retrievers is feasible in the treatment of vertebrobasilar occlusion. These initial results must be confirmed by further prospective studies with a larger number of patients.

\section{REFERENCES}

1. Archer CR, Horenstein S. Basilar artery occlusion: clinical and radiological correlation. Stroke 1977;8:383-90

2. Kim HY, Chung CS, Moon SY, et al. Complete nonvisualization of the basilar artery on MRF angiography in patients with vertebrobasilar ischemic stroke: favourable outcome factors. Cerebrovasc Dis 2004;18:269-76

3. Davis SM, Donna GA. Basilar artery thrombosis: recanalization is the key. Stroke 2006;37:2440

4. Randomised controlled trial of streptokinase, aspirin, and combination of both in treatment of acute ischemic stroke: Multicentre Acute Stroke Trial-Italy (MAST-I) Group. Lancet 1995;346:1509-14

5. Thrombolytic therapy with streptokinase in acute ischemic stroke: the Multicentre Acute Stroke Trial-Europe Study Group. N Engl J Med 1996;335:145-50

6. The International Stroke Trial (IST): a randomised trial of aspirin, subcutaneous heparin, both, or neither among 19435 patients with acute ischemic stroke-International Stroke Trial Collaborative Group. Lancet 1997;349:1569-81

7. Bhatia R, Hill MD, Shobha N, et al. Low rates of acute recanalization with intravenous recombinant tissue plasminogen activator in ischemic stroke: real-world experience and a call for action. Stroke 2010;41:2254-58

8. Lindsberg P, Mattle H. Therapy of basilar artery occlusion: a systematic analysis comparing intra-arterial and intravenous thrombolysis. Stroke 2006;37:922-28

9. Schulte-Altedorneburg G, Hamman GF, Mull M, et al. Outcome of acute vertebrobasilar occlusions treated with intra-arterial fibrinolysis in 180 patients. AJNR Am J Neuroradiol 2006;27:2042-47

10. Sairanen T, Strbian D, Soinne L, et al. Intravenous thrombolysis of basilar artery occlusion: predictors of recanalization and outcome. Stroke 2011;42:2175-79

11. Sarikaya H, Arnold M, Engelter ST, et al. Outcomes of intravenous 
thrombolysis in posterior versus anterior circulation stroke. Stroke 2011;42:2498-502

12. Ellis JA, Youngerman BE, Higashida RT, et al. Endovascular treatment strategies for acute ischemic stroke. Int J Stroke 2011;6:511-22

13. Samaniego EA, Dabus G, Linfante I. Stenting in the treatment of acute ischemic stroke: literature review. Front Neurol 2011;2:76

14. Castaño C, Dorado L, Guerrero C, et al. Mechanical thrombectomy with the Solitaire $A B$ device in large artery occlusions of the anterior circulation: a pilot study. Stroke 2010;41:1836-40

15. Roth C, Papanagiotou P, Behnke S, et al. Stent-assisted mechanical recanalization for treatment of acute intracerebral artery occlusions. Stroke 2010;41:2559-67

16. Costalat V, Machi P, Lobotesis K, et al. Rescue, combined, and standalone thrombectomy in the management of large vessel occlusion stroke using the Solitaire device: a prospective 50-patient singlecenter study—timing, safety and efficacy. Stroke 2011;42:1929-35

17. Machi P, Costalat V, Lobotesis K, et al. Solitaire FR thrombectomy system: immediate results in $\mathbf{5 6}$ consecutive acute ischemic stroke patients. J Neurointerv Surg 2012;4:62-66

18. Miteff F, Faulder KC, Goh AC, et al. Mechanical thrombectomy with a self-expanding retrievable intracranial stent (Solitaire $\mathrm{AB}$ ): expe- rience in 26 patients with acute cerebral artery occlusion. AJNR Am J Neuroradiol 2011;32:1078-81

19. Lutsep HL, Rymer MM, Nesbit GM. Vertebrobasilar revascularizations rates and outcomes in the MERCI and multi-MERCI trials. $J$ Stroke Cerebrovasc Dis 2008;17:55-57

20. Pfefferkorn T, Holtmannspötter M, Schmidt C, et al. Drip, ship, and retrieve: cooperative recanalization therapy in acute basilar artery occlusion. Stroke 2010;41:722-26

21. Mordasini P, Brekenfeld C, Byrne JV, et al. Technical feasibility and application of mechanical thrombectomy with the Solitaire FR revascularization device in acute basilar artery occlusion. AJNR Am J Neuroradiol 2013;34:159-63

22. Andersson T, Kuntze Söderqvist A, Söderman M, et al. Mechanical thrombectomy as the primary treatment for acute basilar artery occlusion: experience from 5 years of practice. J Neurointerv Surg 2013;5:221-25

23. Adams HP Jr, del Zoppo G, Alberts MJ, et al. Guidelines for the early management of adults with ischemic stroke. Stroke 2007;38:1655-711

24. Adams HP Jr, Bendixen BH, Kappelle LJ, et al. Classification of subtype of acute ischemic stroke: definitions for use in a multicenter clinical trial-TOAST. Trial of Org 10172 in Acute Stroke Treatment. Stroke 1993;24:35-41 